\title{
Electron Microscopy of Synthetic Elastomer Latices
}

\author{
Robert R. Stromberg, Max Swerdlow, and John Mandel
}

\begin{abstract}
The effects on the apparent particle size and its frequency distribution arising from the method of specimen preparation, shadoweasting, distortion of shape, and other artifacts inherent in the electron microscopy of synthetic elastomer latices are described. The possibility of applying a single, reproducible method of examination to four latices involving three copolymers (butadiene-styrene, butadiene-acrylonitrile, and butadiene-styrene-acrylonitrile) is statistically evaluated. These copolymers contain particles ranging in size from approximately 100 to $2100 \mathrm{~A}$, and their average particle diameters are about 600, 800, and $1700 \mathrm{~A}$, respectively.
\end{abstract}

\section{Introduction}

\subsection{Purpose and Problem}

The purpose of this investigation is to determine the shape and size of the particles found in a variety of commercial and experimental elastomer latices in order to relate the findings with the use of such latices for the impregnation of leather as a substitute for impregnation by means of polymer solutions $[1]^{1}$ - or by means of in situ polymerization [2].

For a comparison of the behavior of different latices in the impregnation of leather and for a comparison of their particle-size distributions, it was thought that a single, reproducible method common to all of them would be necessary in order to discount the errors and artifacts inherent in any one method. Furthermore, it would be necessary to evaluate the advantages and limitations of the method selected as a means for determining the shape, size, and frequency distribution of these synthetic elastomer latices.

Particle-size studies may be made by the methods of radiation diffraction and scattering, surface adsorption, fractionation, and microscopy. Because of the necessity for data on the shape and mode of flocculation, as well as the particle-size distributions of impure synthetic elastomer suspensions, microscopy was chosen. Inasmuch as the size of material lies in the colloidal range, the electron microscope was needed to provide the direct means for observation.

The available methods of specimen preparation impose severe limitations on the accuracy of electron microscopical measurements. An inherent difficulty of such methods lies in the nature of the material being examined. With synthetic elastomer latices, the problem is threefold. 1. To maintain the dispersed material as individual particles. 2. To fix the nonrigid particles in their original, presumably spherical, shape without changing their size. 3. To separate the polymer particles from other material in the suspension.

If one applies the usual method of specimen preparation involving direct dilution with water and drying onto a collodion substrate, the particles flatten and merge into a hazy layer in which one particle cannot be distinguished from another. With

1 Figures in brackets indicate the literature references at the end of this paper. further dilution, layers of elastomer of smaller area are formed, but still no particulate structure is exhibited.

\subsection{Evaluation of Existing Methods}

Two approaches have been used to overcome some of these difficulties. In one, the latex was dispersed in an aqueous solution of polyvinyl alcohol; and upon drying, the particles became embedded in the resulting plastic film [3]. It was found that the tendency of the particles to flatten and coalesce was reduced. However, this technique impairs image sharpness and contrast because of additional scattering and reduced transmission of the electron beam. Shadowcasting such a specimen is not effective and, therefore, observations of the third dimension and the extent of flattening of the particles are precluded. Also, any extraneous matter, such as emulsifying agent, catalyst, inhibitor, etc., in the original latex suspension will contaminate and partially obscure the elastomer particles in the preparation.

In the other approach, the latex suspension was brominated [4]. This served to diminish the amount of flattening by hardening the soft particles. Application of bromine by itself may fix the particles as spheres, but severe flocculation prevents accurate particle-size counts. The use of a commercial wetting agent, Daxad-11 [5] or Aerosol OT [6], as a dispersing agent in addition to bromination has been described. Although the dispersing agent reduces flocculation, the excessive amount required encases and obscures the individual elastomer particles. To remove the excess dispersant a thorough washing of the final specimen preparation is necessary.

Neither the exact washing technique nor the effect of washing on the particle-size distribution have been described. Regarding the shape of the particles, assumptions have been made that the particles are either perfect spheres [4] or ellipsoids [5] resting entirely on top of the sagging substrate. The effect of bromination on the size of the particle has been estimated to be about a 9-percent increase in diameter [4]. A 9.6-percent increase, calculated from an indirect measurement, has also been reported [7]. No correction has been made, however, for partial embedding in the softened substrate. This could be a considerable factor in the case of particle diameters nearly equal to the thickness of the substrate. 


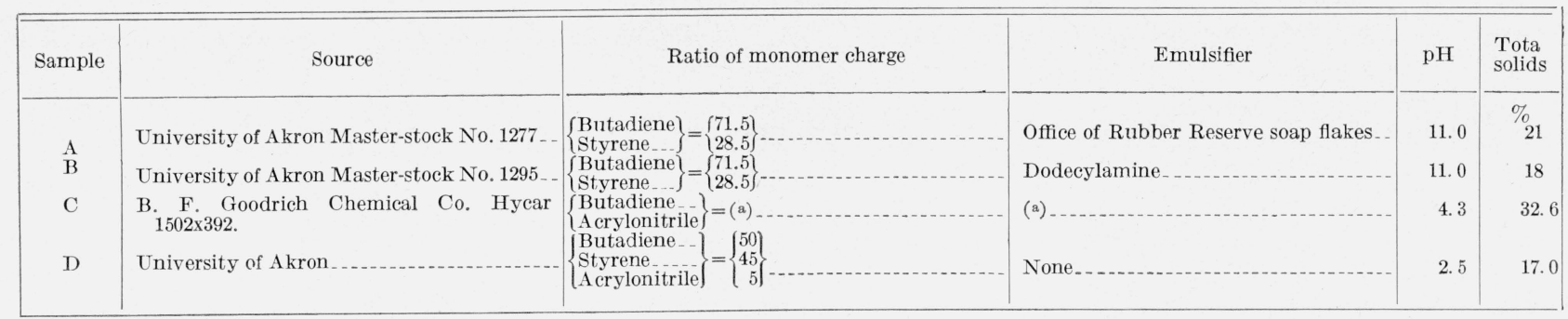

\& Information unavailable.

\section{Materials Used}

The identification and composition of the four latices investigated are contained in the above tabulation $[8,9,10]$.

\section{Specimen Preparation}

\subsection{Exploratory Methods}

Various methods were investigated in attempts to eliminate swelling the particles by bromination and using a dispersing agent, yet fixing their original shape and size and retaining discrete separations between particles. A freeze-drying method was tried, in which a drop of a dilute aqueous suspension of latex was placed upon a collodion-covered specimen screen, supported by a glass microscope slide. The glass slide was immediately placed on a brass block, previously chilled to about minus $100^{\circ} \mathrm{C}$, freezing the drop. The entire block of frozen specimens was placed in the chamber of an RCA type EMV-1 vacuum unit where the ice sublimed during evacuation, leaving the particles on the collodion film.

Another method was tried with latices A and B, in which the $\mathrm{pH}$ of the diluent was matched by addition of ammonia to that of the latex suspension and the diluted suspension was dried onto the collodion substrate.

In a third approach, diluted suspensions were sprayed by means of an ordinary nasal atomizer onto collodion-covered specimen screens. Three or four "shots" of the diluted latex were applied to each screen from a distance of about $1 \mathrm{~m}$.

Each of these methods of specimen preparation frequently gave well-dispersed, round particles with some of the latices, but not consistently with all. Because one reproducible method that was equally good for all the latices was desired, these approaches to the problem of specimen preparation were set aside, even though each method tried showed promise of producing a good specimen for particlesize studies with the electron microscope.

\subsection{Method Used}

In order to arrive at a single, reproducible method suitable for a statistical determination of the particlesize distributions of the four synthetic elastomer latices it was, therefore, decided to apply a combina- tion of various methods already reported. Because such a large quantity of dispersing agent is required to prevent flocculation of the particles and such a thorough washing of the dried specimens is necessary in order to expose the particles as individual units, concern was felt about the preferential removal of certain size ranges. Consequently, it was necessary to devise several specific washing techniques and to evaluate not only the comparative effects of the washing techniques on the particle-size distribution but also the inherent limitations of electron microscopy as a means for determining the size and shape of these latex particles.

A sample of each of the four latices was diluted with an aqueous solution of 0.2 percent of dispersing agent (Daxad-11) to make a rubber concentration of approximately 0.01 percent by weight. A few drops of this diluted suspension were placed in a watch glass and exposed to bromine vapors poured from a bottle of liquid bromine for 15 sec. The brominated suspension was further diluted with distilled water to give a rubber concentration of about 0.0001 percent. Drops of the final suspension were placed upon collodion-covered specimen screens, supported by glass microscope slides, and dried for an hour longer than the time required for the drops to evaporate in an air-conditioned room kept at about $22^{\circ} \mathrm{C}$ and 50-percent relative humidity. Immediately thereafter, each of the following washing techniques was applied to eight separate specimens of each sample of latex. The washing was performed either with distilled water at about $80^{\circ} \mathrm{C}$ or with steam at atmospheric pressure.

\section{a. Jet Washing}

A fine stream of hot water from a wash bottle was played over the prepared specimen screens on the glass slides. The slides were held, specimens up, at an angle of about $45^{\circ}$ from the vertical, and the jet of water was applied at a near-grazing angle. This washing was repeated 3 or 4 times for periods of about 15 -sec duration.

\section{b. Immersion Washing}

The prepared specimen screens supported by the glass microscope slide were immersed edgewise at an angle of about $45^{\circ}$ into a dish of hot water for about 15 sec. Successive immersions and withdrawals were repeated 3 or 4 times in the same dish of water.! 


\section{c. Pipette Washing}

Drops of hot water were placed upon the prepared specimen screens and removed after 15 -sec contact by means of a medicine dropper. This process was immediately repeated 3 or 4 times with fresh drops of water.

\section{d. Steam Washing}

The prepared screens on their supporting glass slides were placed at an angle of about $15^{\circ}$ from the vertical in a wire basket in the upper part of an insulated glass column about $40 \mathrm{~cm}$ long. Steam was passed continuously through the column and around the specimens for a period of about $15 \mathrm{~min}$.

\section{e. No Washing}

One group of specimens from latex sample A was prepared with Daxad-11 and fixed with bromine in the manner described above, except that a slightly lower concentration of rubber was used. Even though no washing technique was applied, an acceptable preparation was obtained (fig. 1, A). Although this unwashed preparation was not reproducible, the micrographs can be used for comparison with the other washing procedures used in the specimen preparations for latex sample $\mathrm{A}$.

\subsection{Shadowcasting}

The specimens were washed and then dried overnight. Those specimens selected for shadowcasting were evacuated to a pressure of about $10^{-5} \mathrm{~mm}$ of mercury or less. Pure electrolytic chromium metal pieces were slowly evaporated from a conical tungsten wire basket placed in such a manner as to create radiation shadows whose lengths were 4 and 5 times the altitude of the particle causing them.

\section{Interpretation of Electron Micrographs}

Figure 1, B, illustrates the appearance and shape of the latex particles when the latex (sample A) is merely diluted with water and allowed to dry out on the collodion substrate. This particular field offers more information than the usual micrograph obtained from such a preparation. The rubber latex is generally coagulated into large flattened masses showing no particulate detail at all. Figure 1, C, is an example of the same latex in which the diluted sample was given additional treatment by exposure to bromine and allowed to dry. Instead of formless masses, round particles are observed. However, the poor dispersion of these discrete particles prevents an accurate measure of their size and number.

Figure 2 represents electron micrographs of latex sample B prepared as described in section 3.2. For figure $2, \mathrm{~A}$, the specimen was given a jet washing; 2, B, immersion; 2, C, pipette; and 2, D, steam. The presence of residual material in the background of these micrographs indicates that the specimens were not overwashed, and that only a minimum number of particles was removed. Excessive wash- ing will remove not only the extraneous material but also a much greater number of particles. The delicacy of the steam washing is shown in figures $1, \mathrm{D}$, and 2, D, where the covering of dispersing agent has not been completely removed. The existence of such an envelope around the particles is borne out by their diffuse outline and by the shortened lengths of the shadows, which would have been five times the diameter for unembedded hard spheres. The envelope of dispersant provides an advantage in that it reduces the free energy of the surface of the water helping to prevent the particles from coagulating during the drying-out process and, in addition, cements them to the collodion substrate. Originally it was believed that steam as a method of washing would prove to be the best, in that it would dissolve away the dispersant leaving all the particles behind. In practice, however, in order to accomplish adequate washing, it was found necessary to allow the steam to condense so that the actual washing was done in part by vapor and in part by hot water. Attempts to prevent condensation of droplets on the specimen preparation by employing additional insulation around the glass column and infrared radiation for the time required for effective washing destroyed the collodion film.

The electron micrographs seen in figure 3 represent the results obtained when the four different latices were given the same washing technique, that is, pipette. The conspicuous differences in the number and the sizes of particles should not be taken to mean that these differences result from preferential removal of particles in the washing process. Rather, they are most likely due to variations in the original concentrations, $\mathrm{pH}$, surface charges, rate of drying, the ratio of the substrate area to the volume of the drop of latex suspension, and other factors that influence the settling out of the particles onto the collodion substrate. No single selected view is necessarily typical of the over-all observations, and a statistical analysis is required before any conclusions can be made with respect to particle-size distributions.

Prior to determinations of the particle size for these latices, a consideration of the actual shape has to be made. The large sphere seen in figure 4 (presumably a mass of molten chromium that fell on the dried collodion substrate and subsequently shadowed) demonstrates what a rigid sphere lying on top of the film would look like when shadowcast in such a way that the length of the shadow is four times that of the height of the particle. Actual measurement gives a value of $9000 \mathrm{~A}$ for the transverse diameter of the "sphere."

(The transverse diameter is that distance measured across the particle and normal to the direction of shadowing.) This value is about 5 percent less than the height determined from the geometry of the shadowcasting technique. This discrepancy can can result from sagging of the supporting substrate under load and a very slight embedding of the particle in the film, as shown schematically in figure 5. Furthermore, the inherent approximations 

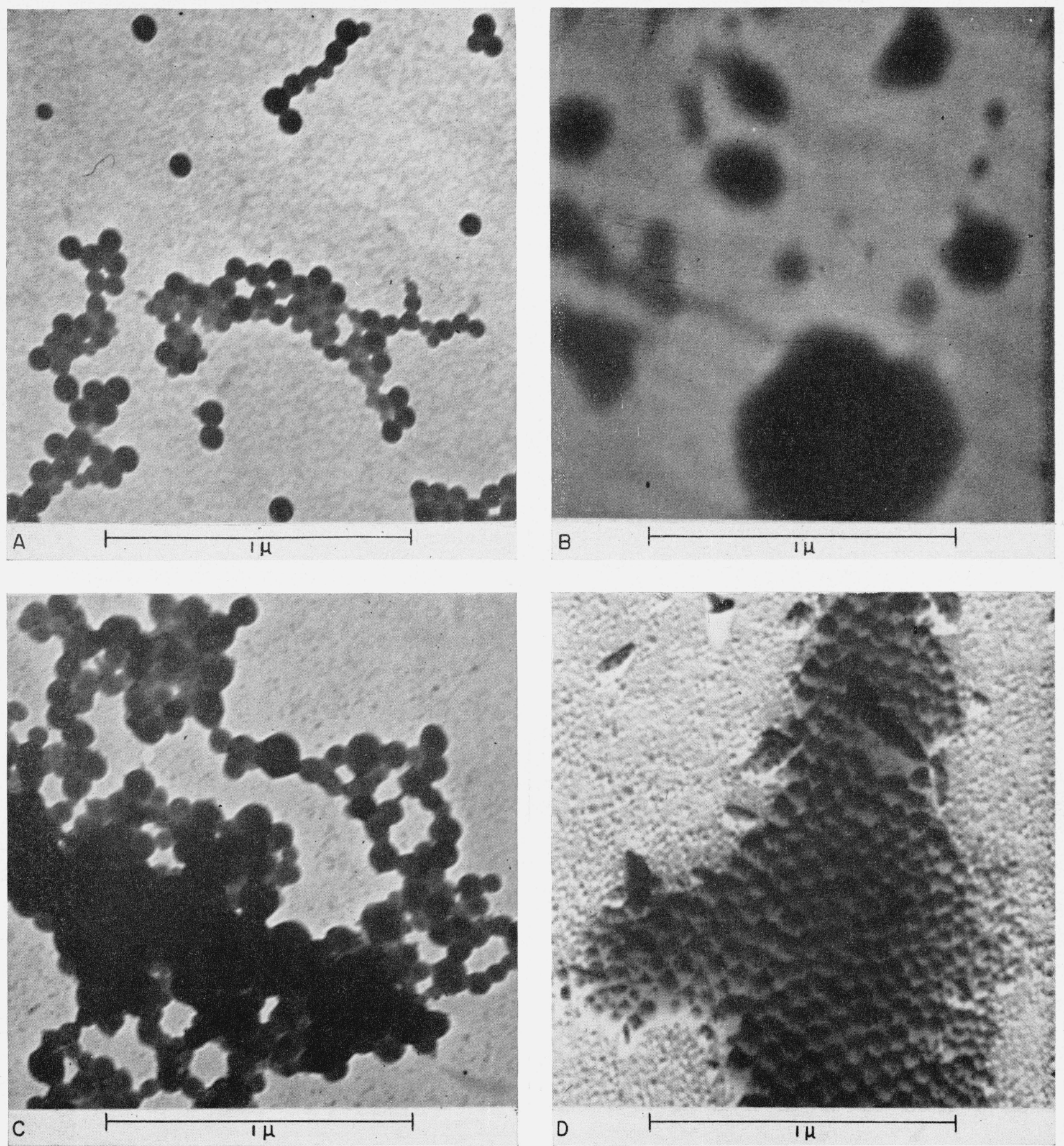

FIGURE 1. Effect of various techniques of specimen preparation on shape and dispersion of latex particles.

A sample A (butadiene-styrene), treated with dispersing agent and bromine but unwashed.

C, sample A (butadiene-styrene), treated with bromine only.
B, sample A (butadiene-styrene), untreated except for dilution.

$\mathrm{D}$, sample C (butadiene-acrvlonitrile), treated with dispersing agent and bromine, steam washed, shadowed 4:1. 


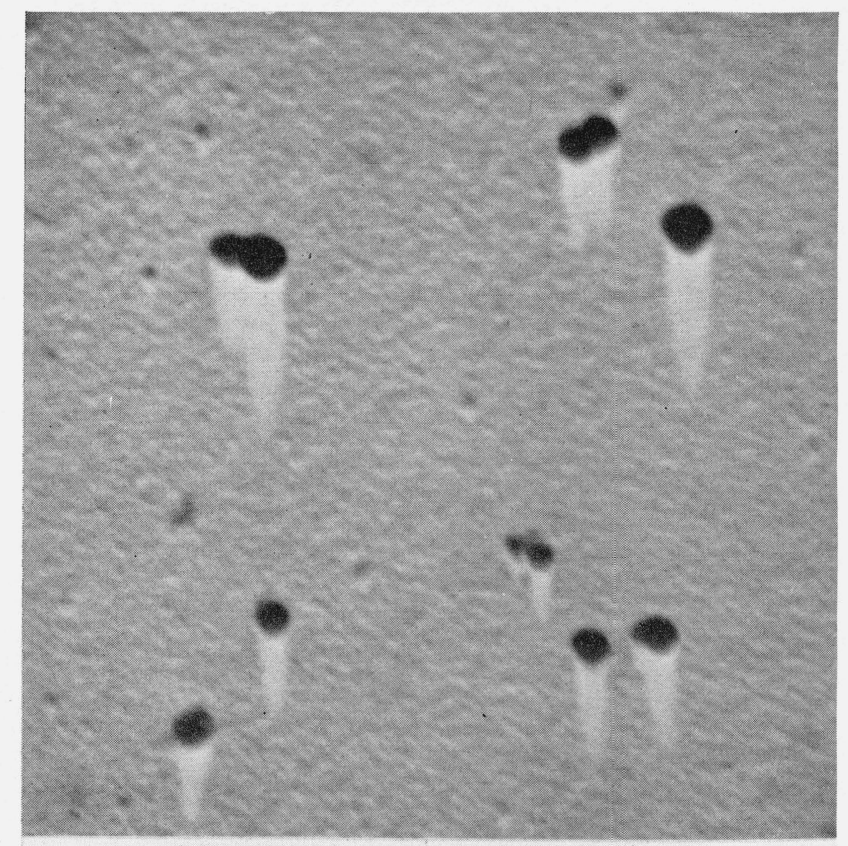

A

$1 \mathrm{H}$

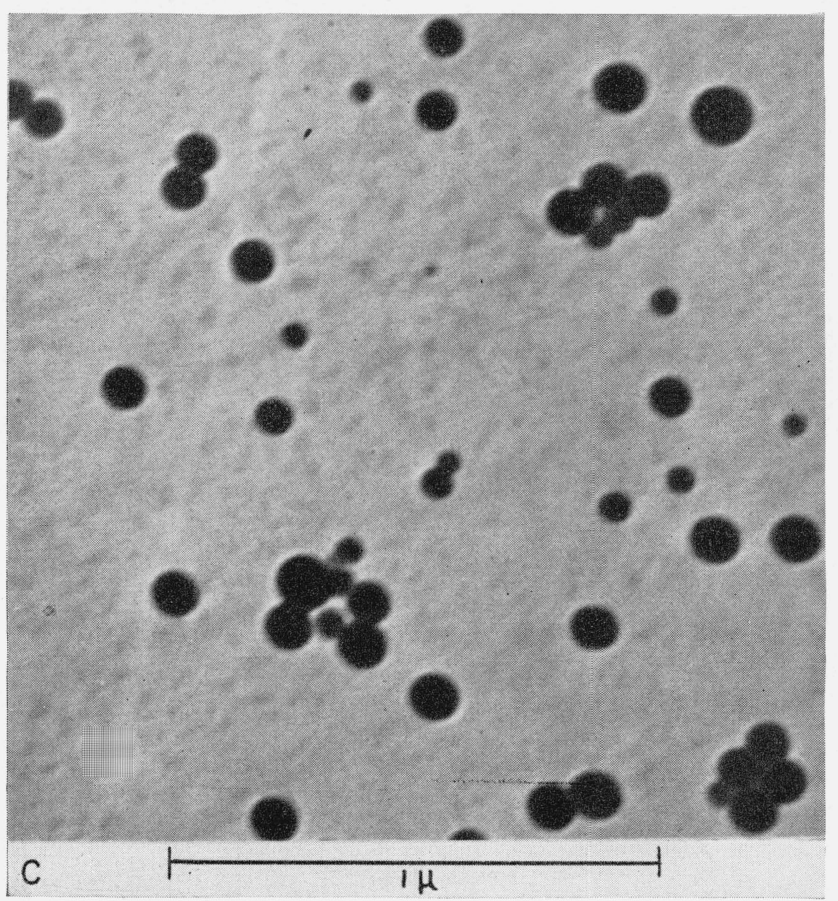

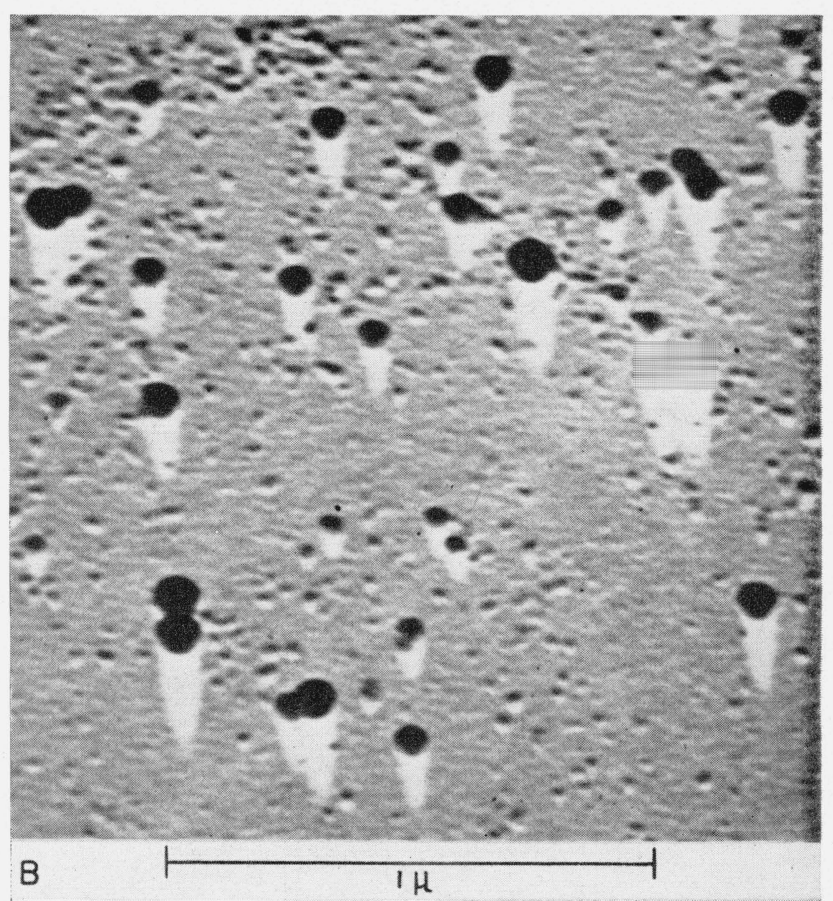

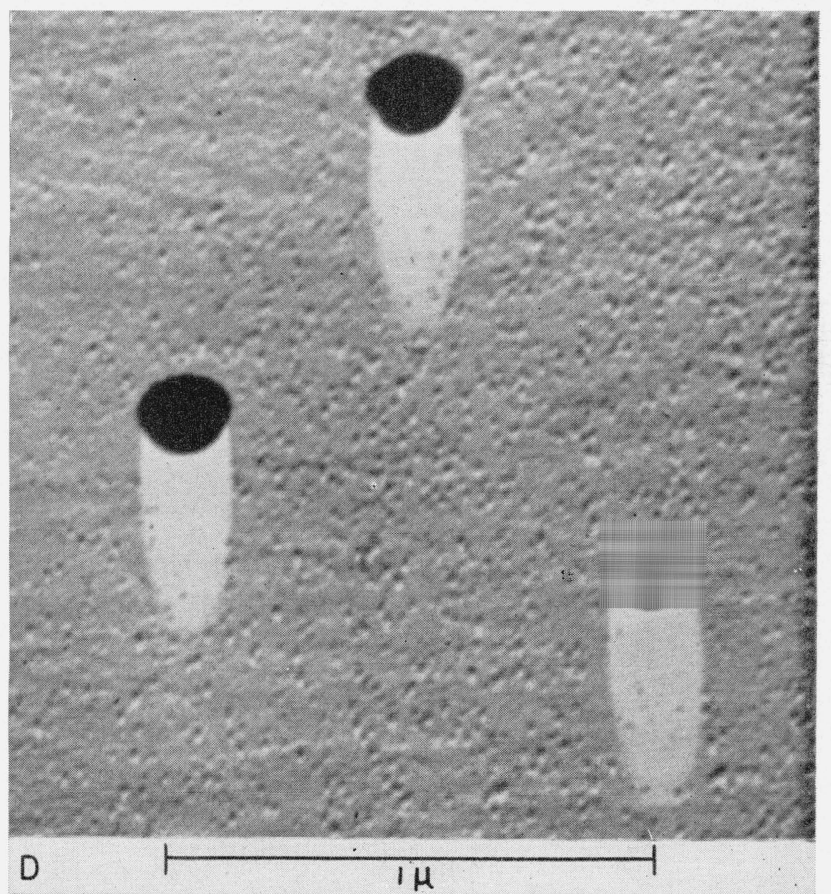

FIGURE 3. One washing technique applied to four latices.

A, sample A (butadiene-styrene), pipette washed, shadowed 5:1.

C, sample C (butadiene-acrylonitrile), pipette washed.
B, sample B (butadiene-styrene), pipette washed, shadowed 5:1.

D, sample D (butadiene-styrene-acrylonitrile), pipette washed, shadowed 4:1. 


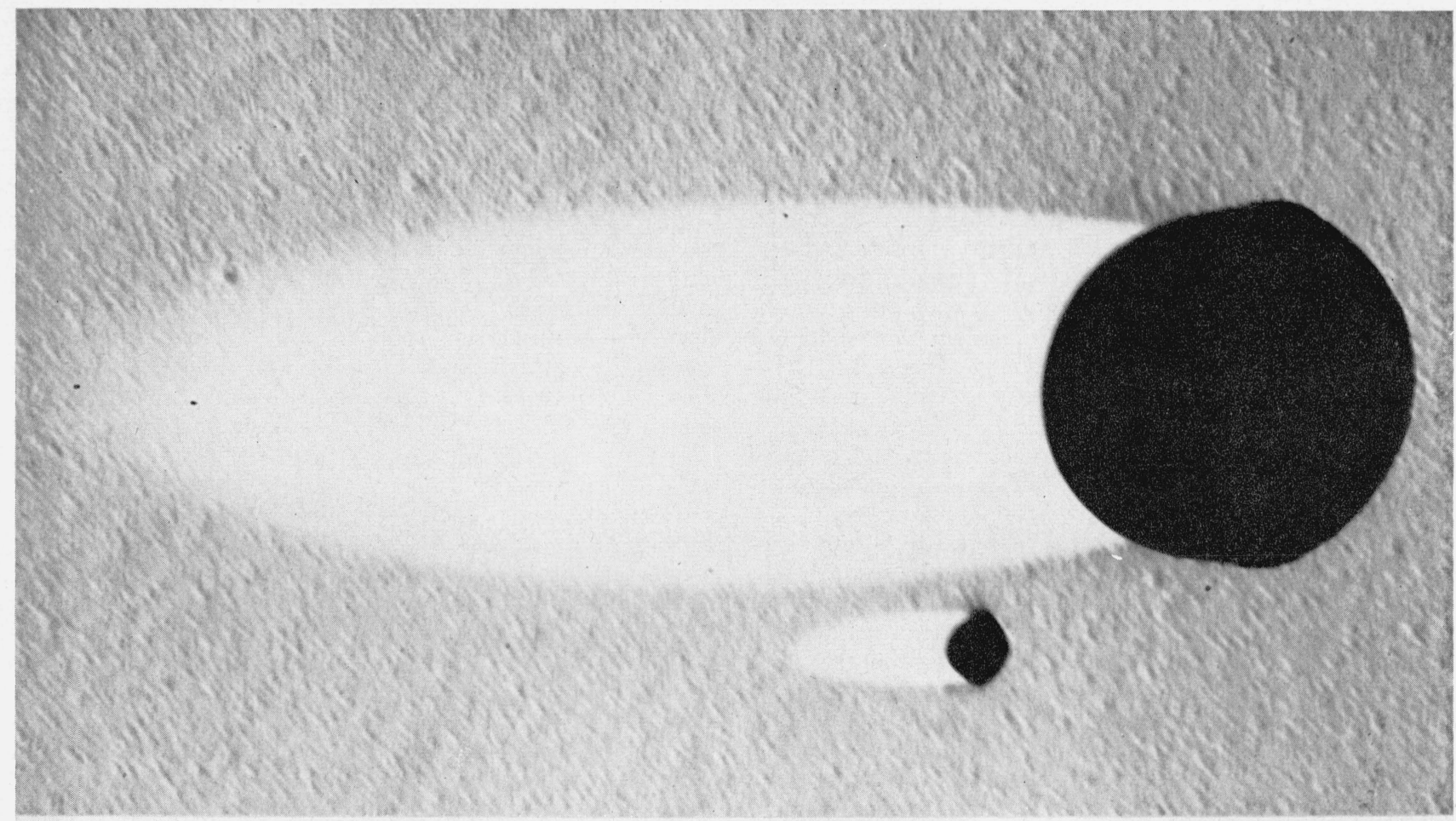

I $\mu$

Figure 4. Sample D (butadiene-styrene-acrylonitrile), jet washed, shadowed 4:1.

The large particle is probably a "sphere" of chromium metal produced at the beginning of the shadowcasting process and subsequently shadowed.

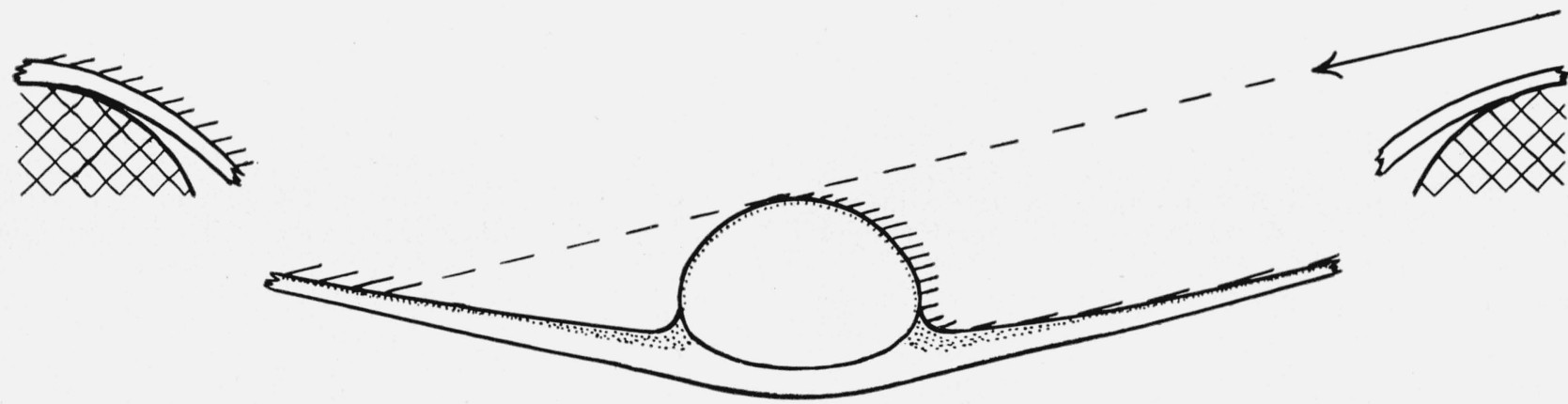

FIGURE 5. Schematic representation of the acquired shape of an elastomer particle.

This shows a cross-sectional view of a single elastomer particle and its probable geometry after treatment with bromine and dispersing agent, and after application of washing and shadowcasting techniques. The'collodion film is shown to sag between the supporting wires of the 200-mesh screen. The particle showning the center produces additional sag and embeds itself into the softened substrate. The dotted area surrounding the particle represents a residual layer of dispersing agent and other material in the latex suspension. Bromination does not harden the particle sufficiently, and the subsequent slumping of the sphere gives it a flattened ovoid shape, Although the shadoweasting indicated by the arrow and dashes, produces a three-dimensional aspect these artifacts, in general, make the use of shadowcasting geometry inaccurate as a means for calculating the diameter of the particle. The observed diameter used in this study is that distance measured across the particle and normal to the direction of shadowing. 
and assumptions made in the setup required for shadowcasting also contribute to this error. If this kind of precision could, nevertheless, be attained on measurements made on the small particles, determination of the diameters derived from the lengths of the shadows would be perfectly valid and well within the experimental errors of the method. Such measurements, however, can be grossly in error because during the removal of the matrix of extraneous material surrounding the particles the hot water softens the collodion substrate, not only causing the film to sag between the wires of the 200-mesh supporting screen but also allowing the particles to sink in and partially embed themselves into the gelatinous collodion membrane. In addition, as was seen in the case of steam washing, some of the matrix of extraneous material remains, thereby partially obscuring the particle. A residual film of dispersing agent and other material in the latex suspension remains to form a layer of varying thicknesses on all the prep. arations. This adsorbed layer of additional material can be seen not only on the collodion substrate but on the latex particles as well. In figure 4 compare the smooth profile of the large sphere produced by a molten mass of chromium, which never was in the latex suspension, with the rougher surfaces of the small latex particles to which a layer of extraneous material dispersed in the latex suspension has been added during the drying-out process.

There is also a flattening of the particles themselves, because exposure of the latex suspension to bromine vapors does not completely fix the particle in its original spherical form. (It is assumed that the particle is a true sphere while in the undisturbed elastomer suspension.) This change in shape results from flow of polymer during the drying-out process when the forces of surface tension of the water and interfacial tensions act upon the nonrigid particle. These forces all act in the same direction, reducing the vertical and increasing the horizontal "diameter". The resultant shape of the particle is probably the flattened ovoid form indicated in figure 5. No direct measurements were made of the distortion of the spherical shape, of the thickness of the adhering layer of extraneous material, or of the amount of sag in the collodion substrate.

All of these factors contribute to a foreshortening of the shadow lengths on the substrate. Thus, calculations to determine the true diameter of these latex particles based upon geometrical relationships between shadow length and altitude can lead to serious errors. On the other hand, the widest portion of the shadow can be used as a criterion for measuring the diameter of these particles only if the metal radiates in effect from a point source. For the distances used, the conical tungsten basket that serves as a crucible and source for the chromium atoms is relatively large. The "polar cap" of metal built up during the deposition of the chromium goes beyond the equatorial diameter of the particle. (The thickness of the "polar cap" may be reduced by the use of suitable metals with higher atomic number, producing adequate lectron scattering with thinner deposits.) In the case of the much larger 9000-A "sphere", this is not as serious an error. The effect of the "polar caps" on relatively small and large particles can be compared in figure 4. Moreover, comparison of the shapes of the shadows caused by the small particles seen in figures 2 and 3 with that of the large particle of figure 4 makes it clear that the almost triangular shadows associated with the small particles are not characteristic of an exposed sphere in contact with a flat surface.

These cumulative errors preclude the measurement of shadows as a precise means for determining particle diameters of these latices. Although the shadowcasting technique does not perfectly reflect the true shape and size of these elastomer particles, it does, nevertheless, provide a three-dimensional aspect to the electron micrographs and creates sufficient contrast to aid the observer in measuring the transverse (horizontal) diameters of the particles.

From the above considerations regarding swelling of the particle upon exposure to bromine and distortion from the spherical shape during the dryingout process in the specimen preparation, a correction of the observed diameters should be applied. Both of these factors operate to make the apparent diameters too large. Contamination-growth of the particles by electron-beam bombardment was kept to a minimum by the avoidance of long exposures. In the opposite sense, because of the vacuum conditions required for shadowcasting and the actual examination of the specimen in the electron microscope, the particles may undergo a shrinkage through loss of monomer. In view of the indeterminate nature of these errors, no quantitative correction could be applied to the observed data.

\section{Measurement of Particles}

At least 10 micrographs of representative fields of each of the samples for each washing technique were taken at 10,000 diameters. The RCA type EMU-2 $(50 \mathrm{kv}$.) electron microscope was used for this work. Calibration of the instrument was obtained by means of a shadowcast Formvar (polyvinyl formal) replica of a planar 4-in. speculum metal diffraction grating having 20,000 rulings per inch. This method of calibration was reliable within 2 percent. The original negatives of the electron micrographs were projected on a screen to provide further linear enlargement of about 30 times. A 10-cm scale in 0.5-mm graduations on a glass plate was simultaneously projected with the electron micrograph. At the magnifications used, the smallest scale division represented $500 \mathrm{~A}$. A pair of dividers was used to compare the diameters of the particles with the superimposed scale. Some of the extraneous material appeared in the final preparation as discrete particles less than $100 \mathrm{~A}$ in size. In order to avoid confusion with elastomer particles, an arbitrary lower limit was set. Consequently, no particles under $100 \mathrm{~A}$ were counted. The diameters were measured to the nearest $100 \mathrm{~A}$. The values reported here are the apparent diameters of the particles as observed in the projected electron micrographs. 


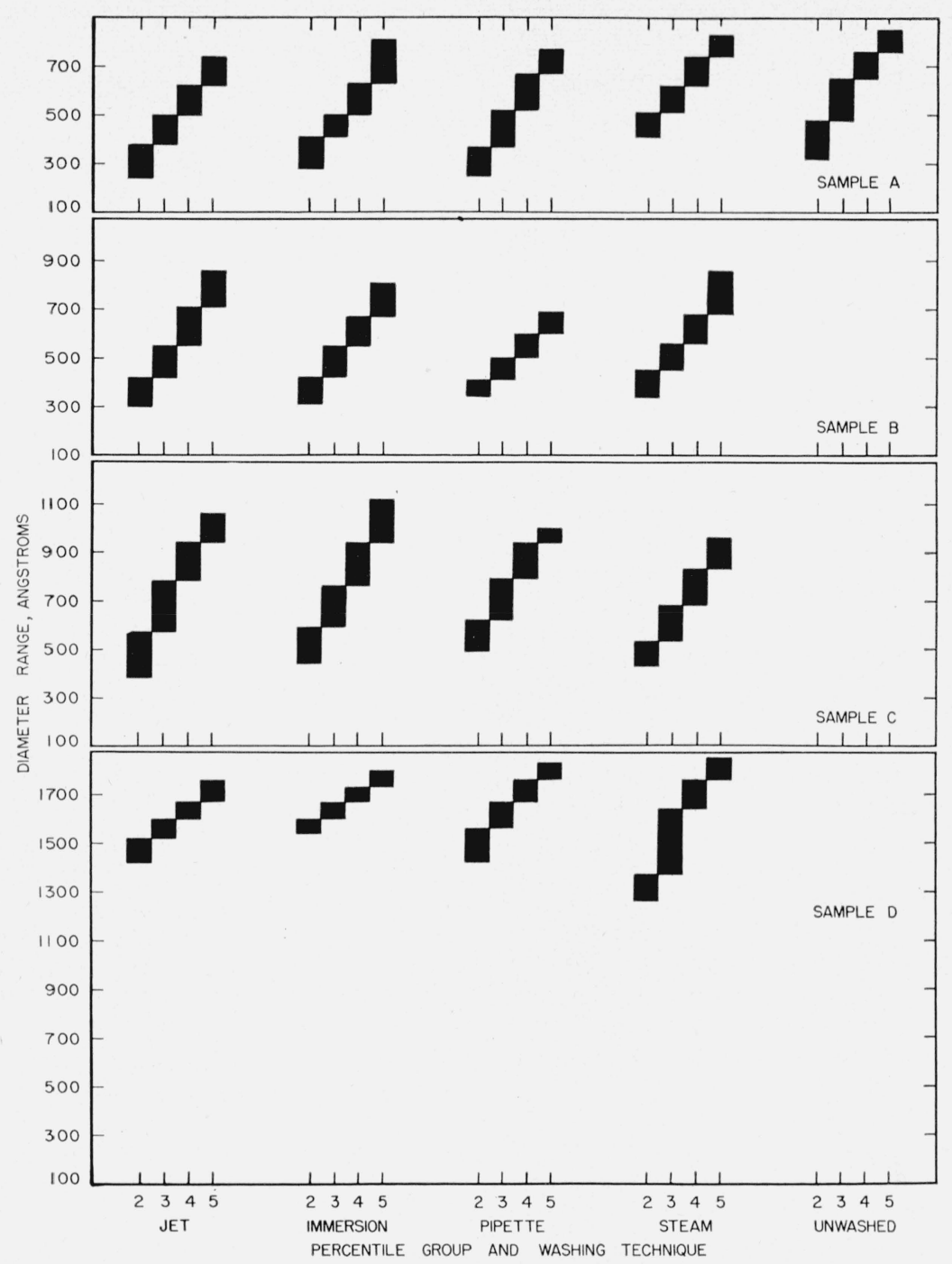

FIGURE 6. Effect of washing techniques on particle-size distribution of synthetic elastomer latices.

Percentile group: $2=10$ to $25,3=25$ to $50,4=50$ to 75 , and $5=75$ to 90 .

\section{Statistical Data and Their Physical Significance}

A comparison of average particle diameters obtained from individual micrographs of unshadowed and shadowed preparations showed no significant differences. No distinction was made, therefore, regarding this particular feature of the specimen preparation. A statistical analysis [11] was made from the viewpoint of determining what effects, if any, the washing techniques have on the particle size distribution. The results are indicated in figure 6 . The frequency distribution of particle sizes corresponding to each washing technique is summarized for each sample in the following manner. The particles of each size, as observed in the various micrographs corresponding to a particular sample and washing technique, were counted and arranged according to size into six groups. The first group consisted of the smallest particles comprising 10 percent of the total count; the second group of the next larger 15 percent; the third and fourth groups of 25 percent each; the fifth group of the next larger 15 percent; and the sixth group consisted of the largest particles making up the final 10 percent of the total count. The range of particle sizes covered by each of these percentile groups is plotted versus the corresponding group number. The median value occurs at the boundary of the third and fourth percentile groups. Following the usual procedure, it was considered advisable to omit from figure 6 the smallest and highest percentile groups, because of the considerable variability that exists at the extremes for samples of moderate size. The distribution corresponding to the unwashed specimen of sample $A$ is plotted in the same manner in order to provide a comparison with the washed specimens of sample A. 
TABLE 1. Diameters and related data of synthetic rubber latex particles

\begin{tabular}{|c|c|c|c|c|c|c|c|c|c|c|c|c|}
\hline \multirow{2}{*}{ Hot-water washing technique } & \multicolumn{6}{|c|}{ Sample A.-Butadiene-styrene } & \multicolumn{6}{|c|}{ Sample B.-Butadiene-styrene } \\
\hline & $m$ & $\bar{x}$ & $s_{\bar{x}}$ & $s_{m}$ & $n$ & $k$ & $m$ & $\bar{x}$ & $s_{\bar{x}}$ & $s_{m}$ & $n$ & $k$ \\
\hline $\begin{array}{l}\text { Jet } \\
\text { Immersion } \\
\text { Pipette } \\
\text { Steam } \\
\text { Unwashed } \\
\text { Sample mean }\end{array}$ & $\begin{array}{ll} & A \\
& 500 \\
500 \\
520 \\
\\
\\
\text { в } 620 \\
650 \\
540\end{array}$ & $\begin{array}{l}\text { A } \\
550 \\
580 \\
570 \\
680 \\
\text { a } 660 \\
600\end{array}$ & $\begin{array}{r}A \\
7 \\
17 \\
12 \\
15 \\
8\end{array}$ & $\begin{array}{c}A \\
60 \\
38 \\
63 \\
27 \\
88 \\
-\end{array}$ & $\begin{array}{r}672 \\
137 \\
283 \\
129 \\
680 \\
-\end{array}$ & $\begin{array}{r}9 \\
5 \\
5 \\
5 \\
10 \\
-\end{array}$ & $\begin{array}{c}A \\
550 \\
550 \\
500 \\
560 \\
540\end{array}$ & \begin{tabular}{c}
$A$ \\
620 \\
610 \\
560 \\
630 \\
\hdashline 600
\end{tabular} & $\begin{array}{r}A \\
12 \\
11 \\
5 \\
10 \\
\end{array}$ & $\begin{array}{r}A \\
104 \\
116 \\
51 \\
110 \\
-\end{array}$ & $\begin{array}{r}329 \\
266 \\
739 \\
355 \\
- \\
-\end{array}$ & $\begin{array}{r}9 \\
10 \\
10 \\
9 \\
- \\
-\end{array}$ \\
\hline 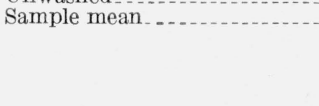 & \multicolumn{6}{|c|}{ Sample C.-Butadiene-acrylonitrile } & \multicolumn{6}{|c|}{ Sample D.-Butadiene-styrene-acrylonitrile } \\
\hline $\begin{array}{l}\text { Jet } \\
\text { Immersion } \\
\text { Pipette } \\
\text { Steam } \\
\text { Sample mean }\end{array}$ & $\begin{array}{l}780 \\
760 \\
790 \\
680 \\
750\end{array}$ & $\begin{array}{l}800 \\
820 \\
820 \\
740 \\
800\end{array}$ & $\begin{array}{r}14 \\
15 \\
8 \\
9\end{array}$ & $\begin{array}{r}214 \\
76 \\
57 \\
141 \\
-\end{array}$ & $\begin{array}{c}314 \\
284 \\
725 \\
618 \\
-\end{array}$ & $\begin{array}{l}10 \\
10 \\
10 \\
10\end{array}$ & $\begin{array}{l}1600 \\
1670 \\
1670 \\
1640 \\
1640\end{array}$ & $\begin{array}{l}1650 \\
1710 \\
1690 \\
1610 \\
1660\end{array}$ & $\begin{array}{r}16 \\
18 \\
25 \\
44 \\
\end{array}$ & $\begin{array}{r}101 \\
91 \\
112 \\
272 \\
\end{array}$ & $\begin{array}{c}78 \\
90 \\
70 \\
49 \\
\end{array}$ & $\begin{array}{l}10 \\
10 \\
10 \\
10 \\
-\end{array}$ \\
\hline
\end{tabular}

a Excluded from sample mean.

Symbol: $m=$ median of particle diameters observed in all micrographs combined, $\bar{x}=$ average of particle diameters observed in all micrographs combined, $s_{\bar{x}}=$ standard deviation of $\bar{x}$,

$s_{m}=$ standard deviation of averages of particle diameters observed in single micrographs, $n=$ total number of particles measured,

$k=$ number of micrographs.

The graphical presentation of these data shows no consistent systematic differences among the four washing techniques with regard to particle-size dis tribution. A more detailed statistical analysis does uncover some significant variation among the distributions corresponding to any one sample, but the lack of consistency of these effects from sample to sample and the smallness of their variations renders them unimportant from a practical point of view. For sample A, the effect of washing can be evaluated with reference to the unwashed sample. Figure 6 shows that this effect, regardless of the washing technique employed, is slight.

Table 1 lists the essential statistical information relating to the particle-size measurements described in this paper. The medians and averages are based on measurements obtained from all the elastomer particles counted. The average values obtained by the various techniques for any given latex do not differ from each other by more than 11 percent for sample B, 9 percent for sample C, and 6 percent for sample $D$. In the case of sample $A$, the discrepancies are somewhat larger (approximately 20 percent) because of errors of an accidental nature that occurred during the preparation. (During the steam washing of this sample the specimens were prematurely removed from the steam bath and insufficiently washed, making the measurement of the particles difficult because of uncertainties regarding their edges.) Judged on the basis of their standard deviations, denoted by the symbol $s_{\bar{x}}$, the averages listed show that any of the four washing techniques could be used to distinguish latices $\mathrm{A}$ and $\mathrm{B}$ from latex $\mathrm{C}$ or $\mathrm{D}$, and latex $\mathrm{C}$ from $\mathrm{D}$.

It is interesting to note that in almost all cases, the median particle size is smaller than the corresponding average value. This would indicate an apparent skewness in the particle-size distribution. Very probably this effect was brought about by the fact that no particles smaller than $100 \mathrm{~A}$ in diameter were recorded. Therefore, the distributions are, in reality, truncated at the lower end, rather than skewed.

An indication of the reproducibility of the experimental procedure may be obtained from the magnitude of the fluctuations of average particle sizes calculated from individual micrographs. For this purpose the values $s_{m}$ were computed. These are standard deviations characterizing the variability of the averages obtained from the single micrographs corresponding to a given latex and washing technique. Thus, the standard deviations $s_{m}$ express the fluctuations of averages of single micrographs, whereas the standard deviations $s_{\bar{x}}$ express the fluctuations of averages based on all particles from the combined total of all micrographs. Accordingly, every $s_{m}$ should be larger than the corresponding standard deviation $s_{\vec{x}}$ by a factor depending on the ratio of the number of particles in a single micrograph to that of all micrographs combined. A statistical examination based on an analysis of the variances shows, however, that in each case the difference between the two standard deviations is larger than can be accounted for by this ratio. This suggests that more variability exists among micrographs than would be inferred from the average range of particle sizes within a single micrograph. (These findings are in line with the observation made in section 4 about the conspicuous differences observed among the individual micrographs of figs. 2 and 3.) Consequently, the advantage gained from the examination of a greater number of micrographs is larger than that due to the mere increase in the number of particles. From the viewpoint of a comparison of washing techniques, it is significant that the additional variability among micrographs is approximately the same for all washing techniques.

On the basis of the foregoing analysis of the data, it appears that each of the four washing techniques is equally suitable and there is little reason for 
selecting any single technique as being the most suitable for all the latices studied here. These latices contained particles ranging in size from approximately 100 to $2100 \mathrm{~A}$. The use of any of the 4 washing techniques resulted in the same classification of the 4 latices into 3 separate groups, with average particle diameters of about 600,800 , and $1700 \mathrm{~A}$, corresponding to the 3 copolymers of butadiene-styrene, butadiene-acrylonitrile, and butadiene-styrene-acrylonitrile, respectively. All the washing techniques yielded results that agreed within approximately 15 percent and had approximately the same order of reproducibility. Consequently, the selection of a single washing technique might be based on practical considerations. Inasmuch as the pipette procedure is experimentally easier to carry out and to control, this technique may tentatively be considered as preferable.

In view of the fact that absolute values for the particle size and frequency distribution of these synthetic latices are not available for comparison, the absolute accuracy of the results obtained by electron microscopy cannot be ascertained. These results do, however, provide the only data available at present and are adequate for reliable intercomparisons among latices.

The practical conclusion that one can draw from this analysis is that although the data do not permit one to make exact quantitative recommendations, they nevertheless provide substantial qualitative and even semiquantitative information regarding the effect of the various techniques of specimen preparation upon the distribution of particle sizes for the electron microscopical examination of the four synthetic latices. With a knowledge of the pore-size distribution of a given leather, the data shown in figure 6 would enable the leather technologist to estimate the number of particles of a given latex that could possibly penetrate the pore structure.
The authors welcome this opportunity to express their indebtedness to several members of the National Bureau of Standards: to Mary N. Steel for assistance with the calculations of the statistical data; and to René Oehler, Samuel G. Weissberg, Chares E. Weir, Norman P. Bekkedahl, and Lawrence $A$. Wood for their suggestions concerning these problems.

\section{References}

[1] R. Oehler, T. J. Kilduff, and S. Dahl, Treatment of leather with castilloa and hevea rubbers, J. Am. Leather Chem. Assoc. 45, 349-77 (1950).

[2] R. Oehler and T. J. Kilduff, Treatment of Leather with synthetic resins, J. Research NBS 42, 63-73 (1949) RP1951.

[3] R. H. Kelsey and E. E. Hanson, A method for preparing rubber latex specimens for the electron microscope, J. Appl. Phys. 17, 675-77 (1946).

[4] W. E. Brown, Procedure for preparing latex samples for electron micrographs, Proceedings of the Electron Microscope Society of America, Abstract 33, J. Appl. Phys. 18, 273 (1947).

[5] S. H. Maron, C. Moore, and A. S. Powell, Electron microscopy of synthetic latices, J. Appl. Phys. 23. 900-5 (1952).

[6] E. Schmidt and R. H. Kelsey, Creaming latex with ammonium alginate, Ind. Eng. Chem 43, 406-12 (1951).

[7] E. A. Willson, J. R. Miller, and E. H. Rowe, Adsorption areas in the soap titration of latex for particle-size measurement, J. Phys. \& Colloid Chem 53, 357-74 (1949).

[8] W. K. Taft, University of Akron, private communication, August 8, 1951. (Samples A and B).

[9] G. A. Daum, B. F. Goodrich Chemical Co., private communication, February 27, 1951. (Sample C).

[10] G. S. Whitby, University of Akron, private communication, February 9, 1951). (Sample D).

[11] S. S. Wilks, Elementary Statistical Analysis, Chapter 2, (Princeton University Press, Princeton, N. J., 1949).

Washington, March 31, 1953. 